\title{
Bulletin Board
}
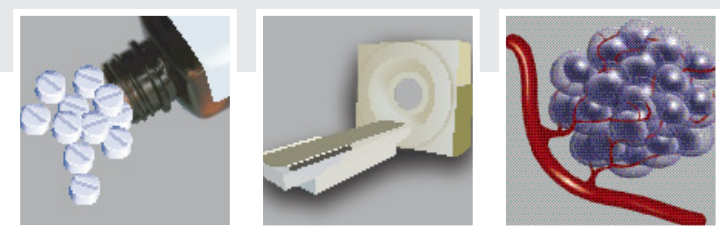

\section{Launch of collaboration to prevent cervical cancer in Rwanda through vaccination and screening}

The Government of Rwanda, Merck (NJ, USA) and QIAGEN (Hilden, Germany) recently announced the launch of Africa's first comprehensive cervical cancer prevention program incorporating both vaccination against human papillomavirus (HPV) and testing for infection with this virus.

Human papillomavirus is linked with cervical cancer and the $\mathrm{WHO}$ reports that it is associated with 288,000 deaths annually, making it the second biggest cause of female cancer mortality worldwide.

The launch, in Kigali, Rwanda, involves vaccination with GARDASIL ${ }^{\circledR}$ (HPV Quadrivalent [types 6, 11, 16 and 18]

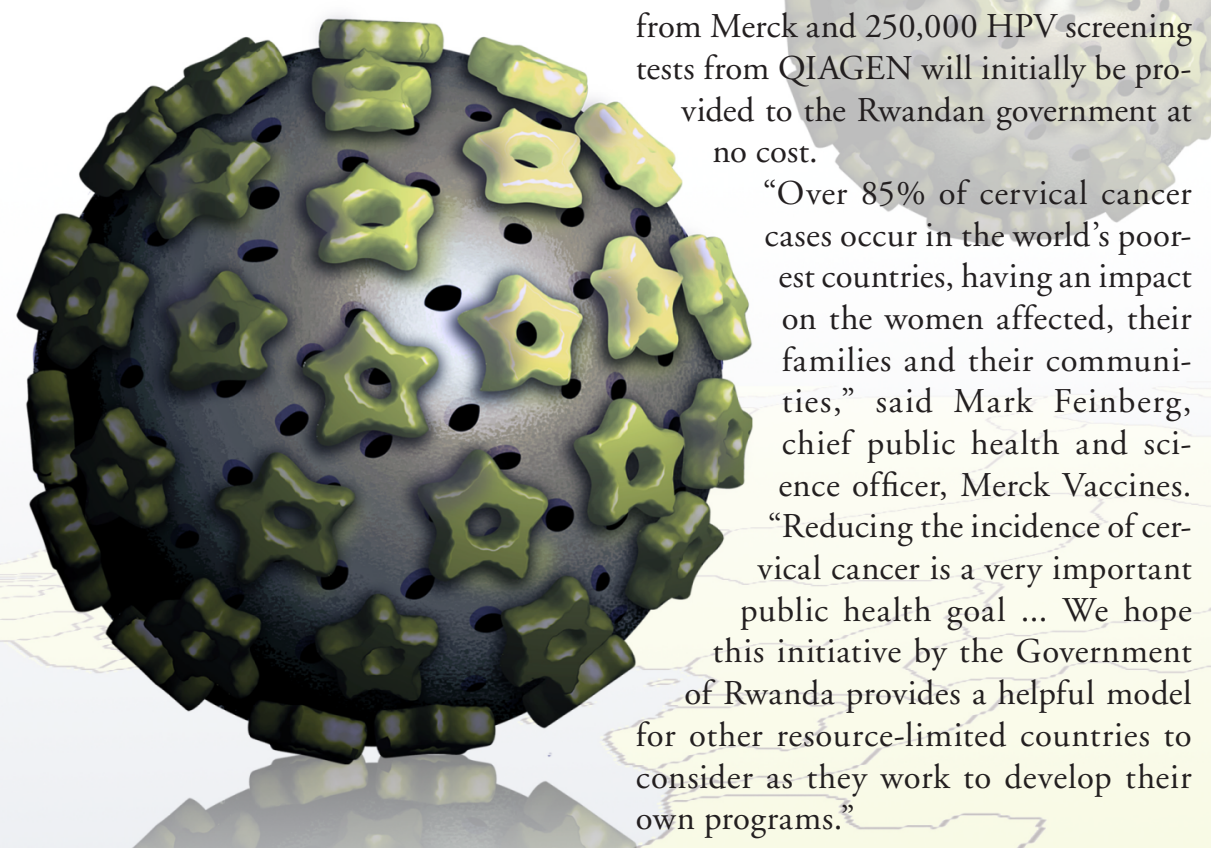

\section{in the news...}

- Lead story: Launch of collaboration to prevent cervical cancer in Rwanda through vaccination and screening

- Man's best friend helping researchers uncover the key genetic players in lymphoma pg 712 girls aged $12-15$ years along with DNAbased molecular diagnostic screening for women of between 35 and 45 years of age.

"It is our goal to create a comprehensive, coordinated program that includes HPV vaccination, cancer screening with HPV DNA testing, and treatment in order to address the nation's unmet needs for cervical cancer-related health services," said Richard Sezibera, Rwanda's Minister of Health, of the program. "This vaccination and screening program brings us one step closer to reaching our goal of protecting the girls and women in our country."

A total of 2 million doses of GARDASIL from Merck and 250,000 HPV screening ests from QIAGEN will initially be proto the Rwandan government at

"Over $85 \%$ of cervical cancer ases occur in the world's poorimpact on the women affected, their families and their communities," said Mark Feinberg, chief public health and science officer, Merck Vaccines. "Reducing the incidence of cervical cancer is a very important public health goal ... We hope this initiative by the Government own programs."

\section{- Priority Paper Alerts pg 712}

- Treasure in the vault: vault nanocapsules provide hope for cancer immunotherapy pg 713

- Topical treatment shows promise for treating myeloma pg 713
Sources: Press release: www.businesswire.com/ news/home/20110425005622/en/Rwanda-MerckQIAGEN-Launch-Africa's-Comprehensive-Cervical WHO: www.who.int/vaccine_research/diseases/ hpv/en 


\section{Man's best friend helping researchers uncover the key genetic players in lymphoma}

It is hoped that the study, recently published online in the journal, Leukemia and Lymphoma, may help to predict and diagnose non-Hodgkin lymphoma (NHL). Identification of the key genes associated with this type of lymphoma has so far eluded researchers owing to the genetic heterogeneity among human populations. Purebred dogs have much less genetic variation so have been identified as a genetic model for lymphoma.

\section{"Using human patient samples, molecular approaches towards a greater understanding of lymphoma are making advances."}

Matthew Breen, one of the study authors, explains why dogs are an ideal model; "Dogs and humans have highly comparable lymphomas at the clinical and pathological level. By looking at aberrant regions of the dog and human genome we can filter out genes that are not in common and so focus in what remains common (i.e., we can see the wood for the trees). By studying lymphoma in the dog, identifying genes of interest and then translating these into the corresponding human genes, we are working with collaborators in human medical centers to determine if these genes are also key players in human NHL." During the study, the team 'genomically recoded' canine NHL data into a 'virtual human' chromosome and surveyed nonrandom aneuploidy in 150 cases of canine NHL.

When asked whether a greater understanding of the genes involved in lymphoma could lead to more accurate diagnostics, Breen replied; "Lymphoma is a heterogeneous set of subtypes. Using human patient samples, molecular approaches towards a greater understanding of lymphoma are making advances. However, such studies tend to identify a large number of genes of interest. Our study revealed that the DNA copy-number aberrations in canine lymphoma are far less complex than in human NHL. This suggests that a lot of the changes we see in human NHL may be 'background noise'. By using the comparative genomic data to refine the molecular features of NHL, we can redefine how diagnostics (and prognostics) are approached."

Breen also commented on the potential clinical implications of his research; "By identifying aberrations in canine lymphoma, we are narrowing the search for the genes that are likely to be the most significant, assuming that both species share a conserved pathogenetic mechanism. We have already identified aberrations that are restricted to specific canine lymphoma subtypes and now are investigating if these also help refine diagnosis of human NHL. In parallel, we have identified genetic changes in canine lymphoma that are associated with response to therapy and so are now evaluating these genes in human NHL patients."

Sources: Thomas R, Motsinger-Reif A, Breen M et al.: Refining tumor-associated aneuploidy through 'genomic recoding' of recurrent DNA copy number aberrations in 150 canine non-Hodgkin lymphomas. Leuk. Lymphoma DOI:10.3109/10428194.2011.559 802 (2011) (Epub ahead of print).

North Carolina State University news release: http://news.ncsu.edu/releases/breen-lymphoma/

\section{Priority Paper Alerts}

Loizou JI, Sancho R, Kanu N et al.: ATMIN is required for maintenance of genomic stability and suppression of B cell lymphoma. Cancer Cell 19(5), 587-600 (2011).

Chromosomal translocations can be initiated by defective rearrangement of immunoglobulin heavy or light chains, or defective class switch recombination. Such chromosomal translocations can be suppressed by the action of the DNA-damage kinase ATM. In the present study, the authors set out to try to uncover the mechanisms of ATM regulation. They found that a cofactor called ATMIN was required for ATM to function correctly. In a mouse model, the group knocked out ATMIN in B cells, a process that resulted in the development of B-cell lymphomas. Further investigation demonstrated that the B cells had defective immunoglobulin rearrangem, ent and class-switch recombination. The authors concluded that their results indicate that ATMIN is required for the efficient repair of DNA breaks produced during somatic recombination.

Bard-Chapeau EA, Li S, Ding J et al.: Ptpn11/Shp2 acts as a tumor suppressor in hepatocellular carcinogensis. Cancer Cell 19(5), 629-639 (2011). 


\section{Treasure in the vault: vault nanocapsules provide hope for cancer immunotherapy}

New research by scientists at the University of California Los Angeles (UCLA [CA, USA]) has demonstrated that 'vault nanocapsules' - nano-sized capsules present in all mammalian cells - could hold promise for cancer immunotherapy.

The study represents an alternative approach in an ongoing effort to use modified autologous dendritic cells (DCs) in cancer immunotherapy. The scientists explored a non-DC-based method, using vault nanoparticles to deliver a protein via injection, into the tumor in order to induce an antitumor immune response in lung cancer.

The long-term, 10-year research project aims to investigate the use of autologous DCs that have been modified to overexpress a secondary lymphoid chemokine (CCL21), a protein involved in the body's immune system. Leonard Rome of UCLA and co-senior author of the project says, "In lung tumors, the immune system is downregulated and what we wanted to do was wake it up, find a way to have the cancer say to the immune system, 'Hey, I'm a tumor and I'm over here. Come get me."'
On administration of the DC's to the tumor site, the overexpression of CCL21 is designed to induce an immune response and cause the body's immune system to target the tumor. This approach has been demonstrated, in a Phase I study and the first in-human study for CCL21, to increase the body's immune response. However, despite these promising results, the preparation and genetic modification of autologous DCs is a costly, technically difficult and time-consuming procedure.

Co-senior author of the paper, Steven Dubinett of UCLA, commented on the approach that was taken to circumvent the disadvantages of the DC approach using nanotechnology: "We thought if we could replace the dendritic cells with a nanovehicle to deliver the CCL21, we would have an easier and less expensive treatment."

The nanoparticles, or vaults, were modified for the slow release of CCL21 into the tumor, results of preclinical studies in a murine lung cancer model demonstrated that the protein-containing vaults stimulated an enduring immune response and that this resulted in attack on cancer cells.

In the next 3 years, the scientists hope their research on the vault-delivery approach progresses to human studies, which would represent a first for vault nanoparticle research. Owing to its slow-release properties, the researchers predict that the vault particle therapy would only need to be administered once. The authors also suggest that the vaults could be functionalized with antibodies, to facilitate tumor targeting, personalized through addition of an individual's tumor antigens, and provide an imaging modality through targeting and fluorescent functional groups, representing an exciting potential vehicle for the future of immune therapy in cancer.

Sources: Kar U, Srivastava M, Andersson A et al.: Novel CCL21-vault nanocapsule intratumoral delivery inhibits lung cancer growth. PLoS ONE 6(5), E18758 (2011).

'I'm a Tumor and I'm Over Here!' Nanovaults Used to Prod Immune System to Fight Cancer. www.sciencedaily.com/releases/2011/05/110503171739.htm

\section{Topical treatment shows promise for treating myeloma}

Researchers from the Penn State College of Medicine (Hershey, Pennsylvania, USA) have identified a topical compound called ISC-4, which may have the potential to prevent myeloma lesion formation.

By inhibition of Akt3, a protein known to play a central role in $70 \%$ of myeloma cases by preventing cell death, topical ISC- 4 was shown to delay or slow down melanocytic lesion or melanoma development in preclinical models. "The Akt3 signaling pathway is deregulated in the majority of melanomas, making it a promising target, which, if inhibited, could correct the apoptotic - or cell death - defect in melanocytic lesions, thereby preventing this disease," explained study invesitgator Gavin Robertson, professor of pharmacology, pathology, dermatology and surgery, and director of Penn State Hershey Melanoma Center.
Previous research had identified naturally occurring Isothiocyantes, commonly found in cruciferous vegetables such as broccoli and brussels sprouts to possess anticancer properties. Unfortunately, these studies revealed a low chemotherapy potency of isothiocyantes on melanoma cells as high concentrations are needed to be effective. By replacing sulfur with selenium, researchers were able to develop a more potent version, known as isoselenocyanates (ISC-4).

In a study that was recently reported in Cancer Prevention Research, it was found that repeated topical application of ISC-4 reduced tumor cell expansion in laboratorygenerated human skin by $80-90 \%$ and decreased tumor development in mouse skin by approximately $80 \%$. The research also demonstrated that the use of the compound is safe.
Surgical excision is currently used to remove melanocytic lesions or to prevent development into more aggressive cancer.

"Topical or localized treatments, such as those we propose for ISC-4, could permit the use of high local concentrations with minimal toxicity and be useful for treating cutaneous lesions not amenable to surgical removal or other currently available approaches... Addition of agents, such as ISC- 4 to sunscreens, body lotions or creams, could have a profound impact on this market for preventing melanoma," concluded Robertson.

Sources: Nguyen N, Sharma A, Nguyen N et al.: Melanoma chemoprevention in skin reconstructs and mouse xenografts using isoselenocyanate-4. Cancer Prev. Res. 4(2), 248-258 (2011).

Medical News Today: www.medicalnewstoday. com/articles/223391.php?nfid $=42951$ 